\title{
Mammalian cell culture density determination using a laser through-beam sensor
}

\author{
Sarah M Rue ${ }^{\star, 1}$, Paul W Anderson ${ }^{1,2}$, Jessica M Miller ${ }^{1}$, Salvatore G Fanale ${ }^{1}$, Jim Y Chang ${ }^{1}$, Scott M \\ Glaser $^{1} \&$ Scott A Lesley ${ }^{1,3}$ \\ ${ }^{1}$ Genomics Institute of the Novartis Research Foundation, San Diego, CA 92121, USA; ${ }^{2}$ Lilly Biotechnology \\ Center, San Diego, CA 92121, USA; ${ }^{3}$ Merck Research Laboratories, Palo Alto, CA 94304, USA
}

BioTechniques 65: 224-226 (October 2018) 10.2144/btn-2018-0059

Keywords: automation $\bullet$ cell counting $\bullet$ cell culture $\bullet$ cell density $\bullet$ laser $\bullet$ mammalian $\bullet$ spectrophotometry

High-throughput protein expression platforms are increasingly used to produce proteins for many applications: to support studies in structure/function, regulation and proteomics, as well as for direct use as potential biotherapeutic agents for medical applications. Here we describe a device that we refer to as the flask density reader (FDR) consisting of a through-beam laser and sensor, and a customized culture flask-

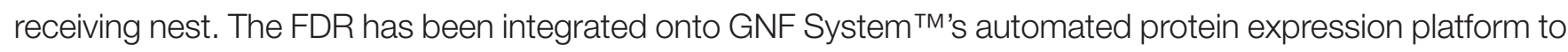
enable rapid, noninvasive, fully automated spectrophotometric determination of cell densities in suspension mammalian cell cultures. The FDR reduces the risk of culture contamination from frequent flask sampling and greatly reduces the time and effort needed to count cells using off-line methods.

Automated workflows for both mammalian transient protein production and protein expression from stable cell lines are used in many laboratories [1,2]. We have previously described fully automated cell culture workflows performed on GNF System ${ }^{\text {TM'S }}$ (CA, USA) protein expression and purification platform (PEPP) for the production and purification of endogenous and recombinant proteins from mammalian cells such as hybridomas, FreeStyle ${ }^{\mathrm{T} M} 293-\mathrm{F}$, (ThermoFisher Scientific \#R79007, MA, USA) and $\mathrm{CHO}$ [3]. On PEPP, cells are maintained in CELLSTAR $^{\circledR}{ }^{\circledR}$ AutoFlasks $^{\text {TM }}$ (Greiner Bio-One \#779190-J, Wein, Austria), which conform to the ANSI SLAS standard footprint compatible with common laboratory automation.

Suspension-adapted cell lines are frequently used for recombinant protein expression from mammalian cells because they grow to high densities and have high cell-specific productivities. Cell lines used on PEPP are suspension-adapted and maintained in shaking incubators. While suspension-adapted lines are easy to handle, cell density and viability must be monitored frequently to follow growth kinetics and to ensure that the culture does not undergo significant cell death prior to harvest. Trypan blue exclusion is a standard assay for determining density and viability of mammalian cells; several companies sell off-line instruments that automate this procedure [4]. While commercially available cell counters confer advantages such as count reproducibility, accuracy and cell viability determination, the user must frequently withdraw samples from the culture vessel. This increases risk of culture contamination and reduces final culture volumes, affecting product yield. Additionally, off-line automated counters typically operate at lower throughput, so as sample numbers increase, more time and effort are spent monitoring cell densities. To address these issues, we worked to develop a high-throughput, noninvasive way to determine cell density. Spectrophotometry is often employed for density determination in bacterial, yeast, algal and human cell cultures [5-7], and we applied that approach here. GNF Systems developed a device called the flask density reader (FDR), which consists of a Keyence Intelligent-B Laser Thrubeam Sensor (Keyence
IB-05) housed in a custom nest designed to hold the AutoFlask (Figure 1). The FDR laser operates at $660 \mathrm{~nm}$ wavelength, and the sensor measures transmitted intensity values for each flask. As the laser beam passes through the AutoFlask ( $1.85 \mathrm{~cm}$ path length), light is absorbed and scattered due to the presence of cells in the culture media. The resulting attenuated light, as detected by the sensor, is inversely related to cell density. The laser beam diameter is $5 \mathrm{~mm}$ (only $0.05 \%$ of the area of the AutoFlask). Like flow cytometry lasers, the FDR laser is rated Class 1, and we have not observed any impact on cells. The FDR is fully integrated onto PEPP and determines cell density in a 500-ms read per AutoFlask.

To interpret FDR output values for $\mathrm{CHO}$ cells, FreeStyle 293-F cells and hybridomas grown in AutoFlasks on PEPP, we generated a standard curve for each cell type by plotting a range of cell culture densities measured on a stand-alone automated cell counter (Beckman Coulter Vi-CELL ${ }^{\text {TM }}$ XR, IN, USA) against FDR output values (Figure 2). Curves were fitted using the nonlinear regression analysis with a two-phase decay equation in GraphPad Prism 7.03. All three resulting

\section{METHOD SUMMARY}

A device called the flask density reader consisting of a through-beam laser and sensor, and a custom cell culture flaskreceiving nest was built and integrated onto an automated protein production platform for rapid, noninvasive, fully automated cell density determination in suspension mammalian cell cultures. 


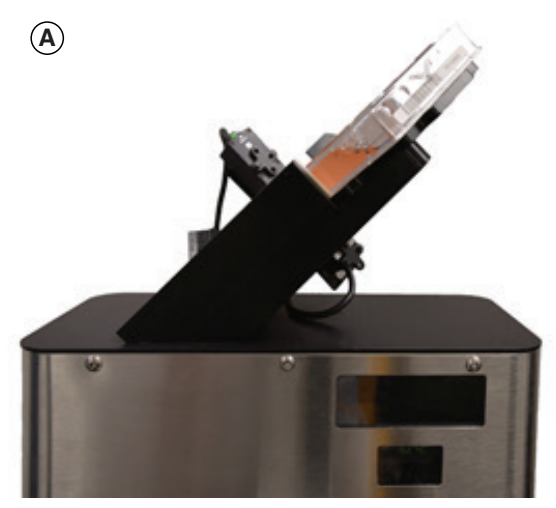

(B)

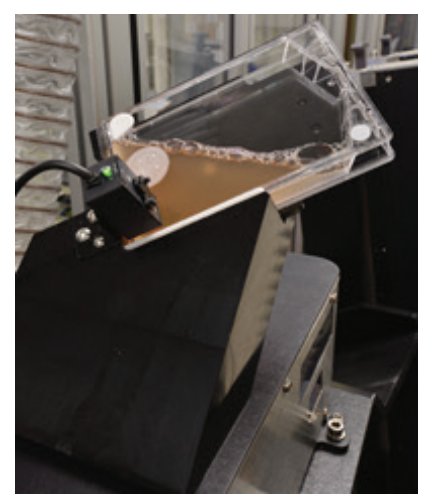

Figure 1. The flask density reader. Front (A) and side (B) views of the flask density reader with an AutoFlask ${ }^{\text {TM }}$ seated in the custom nest.

curves visually yielded satisfactory fits with $R^{2}$ values $>0.99$, and they can be used to interpolate cell densities ( $X$ values) from FDR output ( $Y$ ) values generated for cultures using the Interpolate function in GraphPad Prism (not shown). We recommend that the user establish a standard curve for each combination of cell type, media and labware used to account for factors that could impact FDR output values (e.g., flask material, color changes in phenol red-containing media). GFP expression does not impact FDR reads (not shown).

The FDR can also enable measurement of cell densities in vessels that do not conform to the ANSI SLAS format. For example, we used the FDR for automated determination of cell density in $\mathrm{CHO}$ cultures grown in CELLSTAR T-75 flasks (Greiner Bio-One \#658 175), despite the longer path length $(3.5 \mathrm{~cm}$ ) (Supplementary Figure 1).

Other commercially available noninvasive devices for cell density determination exist, such as those from BugLab (http://buglab.com [8]) and IPRASENSE (www.iprasense.com [9]). The BugLab devices use an infrared or near-infrared light source and multiple detectors or laser/detector pairs, respectively, to provide accurate measurements over a wide range of cell concentrations. The IPRASENSE devices use lens-free microscopy with holographic imaging to determine both cell density and viability. The BugLab devices detect scattered light, so cells can be cultured in virtually any clear vessel, while the FDR detects transmitted light, necessitating the use of vessels with parallel flat surfaces and a defined short path length. The IPRASENSE devices are also built to accommodate vessels with parallel flat surfaces. The noninvasive BugLab devices are designed for either manual operation or surface mounting to a flask or bioreactor with corded data transmission to a base unit, while the FDR and the IPRASENSE devices can accommodate a plurality of flasks, making them more suitable for fully automated, higher-throughput cell density determination.

The FDR cannot be used for cell viability determination, and low cell viability negatively affects FDR data quality (not shown). Due to these limitations, we recommend that the user first monitor both cell density and viability over time using a Vi-CELL ${ }^{\text {TM }}$ or similar method for his/her specific cell line, culture conditions and labware to determine how long high-density cultures can be maintained before a drop in cell viability is observed. The FDR can then be used to monitor cell growth up to that defined density and time-point. Additionally, at FDR output values below 10 (corresponding to $~ 3 e 6$ cells/ $\mathrm{ml}$ for $\mathrm{CHO}$ and hybridoma cultures and $\sim 2.5 \mathrm{e} 6$ cells $/ \mathrm{ml}$ for 293-F cultures), estimated cell densities become less accurate. For all cell lines tested, FDR measurements are not useful at cell densities $<0.1 \mathrm{e} 6 \mathrm{cells} / \mathrm{ml}$. Accordingly, the optimal working range for monitoring $\mathrm{CHO}$ stable pool recovery from selection to determine when pools reach target density for cryo-archiving and inoculating protein production cultures (1e6 cells $/ \mathrm{ml}$ ) is from 0.1 e6 to $~ 3 e 6$ cells $/ \mathrm{ml}$. Hybridoma and 293-F cultures reach lower cell densities than $\mathrm{CHO}$ cultures, so the FDR is also used to monitor growth of these cells.

The FDR can be purchased through GNF Systems (http://gnfsystems.com).

\section{Author contributions}

SR prepared the manuscript with editing by SG. SL and SF conceived of the FDR concept and SF performed proof-ofconcept experiments and designed the device. JC built the FDR and integrated it onto the automated platform. JM, PA and SR generated standard curves using ViCell data and FDR output values.

\section{Competing \& financial interests disclosure}

The authors were all GNF employees at the time of preparation of this manuscript, and this manuscript may help GNF Systems, a company associated with GNF, to sell devices or platforms. The authors have

\section{Guiding New Generations for Decades}

\section{Worthington is supporting life science research and discovery, and we have done so for over 70 years. \\ Authoring technical manuals and guides both in print and digital formats. And, we are cited in thousands of respected scientific journals across the globe.}

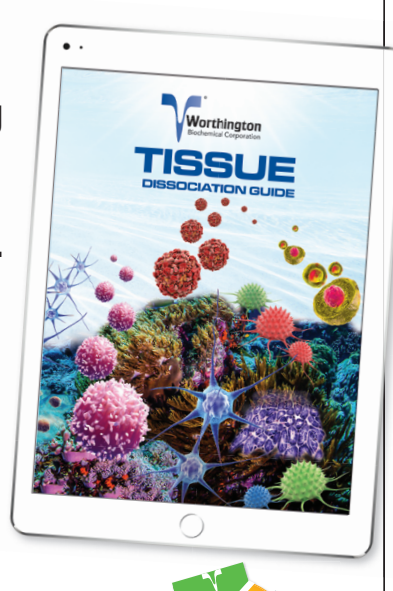

\section{Sharing Our Enzyme Expertise}

Order your free copy of the NEW Tissue Dissociation Guide, 18th Edition. Simply go to:

Worthington-Biochem.com/TissueDissociation/default.html 
(A)

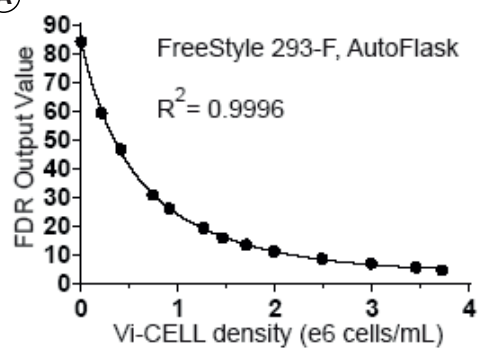

(B)

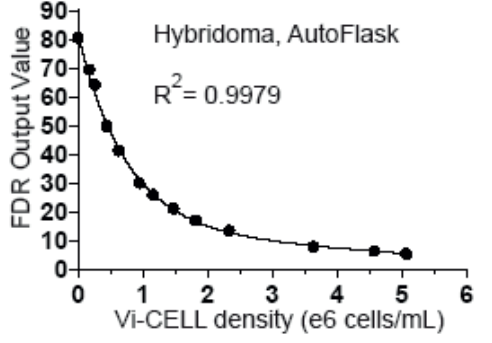

(C)

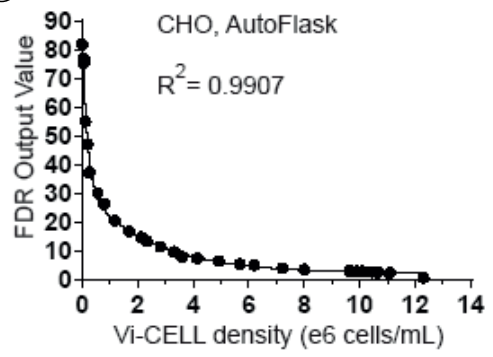

Figure 2. Standard curves for three mammalian cell types grown in AutoFlasks ${ }^{\mathrm{T} m}$. Standard curves created in GraphPad Prism from data generated by reading mammalian cell suspension cultures grown in AutoFlasks side-by-side on the Vi-CELL ${ }^{T M}$ and the flask density reader. (A) FreeStyle ${ }^{\text {TM }}$ 293-F cells, (B) hybridoma cells and (C) $\mathrm{CHO}$ cells.

no other relevant affiliations or financial involvement with any organization or entity with a financial interest in or financial conflict with the subject matter or materials discussed in the manuscript apart from those disclosed.

No writing assistance was utilized in the production of this manuscript.

\section{Open access}

This work is licensed under the Attribution-NonCommercial-NoDerivatives 4.0 Unported License. To view a copy of this license, visit http://creativecommons.org/ licenses/by-nc-nd/4.0/

\section{Supplementary data}

To view the supplementary data that accompany this article please visit the journal website at www.future-science.com/ doi/suppl/10.2144/btn-2018-0059

\section{References}

1. Koehn J, Hunt I. High-throughput protein production (HTPP): a review of enabling technologies to expedite protein production. Methods Mol. Biol. 498, 1-18 (2009).

2. Battle T, Antonsson B, Feger G, Besson D. A high-throughput mammalian protein expression, purification, aliquoting and storage pipeline to assemble a library of the human secretome. Comb. Chem. High Throughput Screen. 9(9), 639-649 (2006).
3. Rue SM, Anderson PW, Gaylord MR, Miller JM, Glaser SM, Lesley SA. A high-throughput system for transient and stable protein production in mammalian cells. In: Methods in Molecular Biology. Vincentelli R (Ed.). (2018).

4. Louis KS, Siegel AC. Cell viability analysis using trypan blue: manual and automated methods. Methods Mol. Biol. 740, 7-12 (2011).

5. Myers JA, Curtis BS, Curtis WR. Improving accuracy of cell and chromophore concentration measurements using optical density. BMC Biophysics 6(1), 4 (2013).

6. Brescia P, Raymond K, Banks P. Monitoring cell growth in $2 \mu$ l volumes using a microplate reader. (2011). www.biotek.com/resources/applicationnotes/monitoring-cell-growth-in-2-I-volumesusing-a-microplate-reader/

7. Ude C, Schmidt-Hager J, Findeis M, John GT, Scheper T, Beutel S. Application of an onlinebiomass sensor in an optical multisensory platform prototype for growth monitoring of biotechnical relevant microorganism and cell lines in single-use shake flasks. Sensors 14(9), 17390-17405 (2014).

8. Debreczeny MP, Romero J, Petersen E. US 2009/0075248 A1 (2009).

9. Allier C, Bordy T, Hervé L et al. Label-free cell viability assay using lens-free microscopy. International Society for Optics and Photonics 10497 (2018). doi:10.1117/12.2289811

First draft submitted: 25 April 2018; Accepted for publication: 7 August 2018

Address correspondence to: Sarah M Rue; Genomics Institute of the Novartis Research Foundation, San Diego, CA 92121, USA; E-mail: srue@gnf.org

To purchase reprints of this article contact: s.cavana@future-science.com 